\title{
siRNA-Act1 Inhibits the Function of IL-17 on Lung Fibroblasts via the NF-KB Pathway
}

\author{
Zhaoxing Dong ${ }^{\mathrm{a}}$ Yanni Yang ${ }^{\mathrm{b}}$ Tao Zhang $^{\mathrm{a}}$ Yongxia Li $^{\mathrm{a}}$ Qinxin Kang ${ }^{\mathrm{a}}$ \\ Wen Lei $^{\mathrm{a}} \mathrm{YuCao}^{\mathrm{a}}$ Xiaoqun Niu ${ }^{\mathrm{a}}$ Dianhua Wang ${ }^{d}$ Wenlin Tai $^{\mathrm{c}}$ \\ ${ }^{a}$ Respiratory Department, ${ }^{b}$ Department of Ophthalmology and ${ }^{c}$ Clinical Laboratory Department, The 2nd Affiliated \\ Hospital of Kunming Medical University, and ${ }^{\mathrm{d} S}$ School of Pharmacy and Yunnan Key Laboratory of Pharmacology for \\ Natural Products, Kunming Medical University, Kunming, China
}

\section{Key Words}

Interleukin-17 · Lung fibrosis · Lung fibroblast · Activator 1

\begin{abstract}
Background: Interleukin (IL)-17-producing T lymphocytes play a role in pulmonary fibrosis, but the possible mechanism of IL-17 on lung fibroblasts remains uncertain. Objectives: To explore the role and possible mechanism of IL-17 on lung fibroblasts. Methods: A mouse model of pulmonary fibrosis was established by intratracheal administration of $5 \mathrm{mg} / \mathrm{kg}$ bleomycin. At 14 days following bleomycin administration the pulmonary fibroblasts were isolated, cultured and identified. siRNA for activator 1 (Act1) were transfected into lung fibroblasts, which were cocultured with IL-17. The NF-KB pathway was detected for IL-17 on the lung fibroblasts. Results: IL-17R was increased significantly at 14 days in the bleomycin-induced pulmonary fibroblast model, exogenous IL-17 significantly promoted the proliferation of the pulmonary fibroblasts in primary culture and obviously increased the expression of a-smooth muscle actin and type I and type III collagen in the fibroblasts. We found that IL-17 rapidly activated the NF- $\mathrm{KB}$ signaling pathway through activated phosphorylated $\mathrm{p} 65$ and $\mathrm{IKB}$, and all roles of IL-17 on lung fibroblasts were inhibited under the interference for the expression of Act1 in lung fibroblasts. Conclusion:
\end{abstract}

IL-17 may directly promote the proliferation, transformation and collagen synthesis of lung fibroblasts via the NF-kB signaling pathway, which can be inhibited by the interference for the expression of Act1.

Copyright $\odot 2013$ S. Karger AG, Basel

\section{Introduction}

Pulmonary fibrosis is histologically characterized by a numerical expansion of fibroblasts and excessive accumulation of extracellular matrix, particularly collagen. The hallmarks of pulmonary fibrosis include cell proliferation, production of extracellular matrix by resident fibroblasts and the presence of immune cells [1]. While not enough is known about the mechanisms responsible for these events, numerous studies have focused on potential mechanisms, including various processes related to inflammation, epithelial-mesenchymal interactions, epithelial apoptosis, endothelial disturbances and coagulation $[2,3]$.

A number of fibroblasts and myofibroblasts are seen during fibrosis, fibroblast migration, proliferation, and

\section{Zhaoxing Dong and Yanni Yang contributed equally to this work.}

\section{KARGER}

E-Mail karger@karger.com

www.karger.com/res
C 2013 S. Karger AG, Basel

0025-7931/13/0864-0332\$38.00/0 
extracellular matrix synthesis and degradation, all of which play important roles in chronic inflammation and fibrosis, and are regulated by various growth factors and cytokines. A key common feature found in fibrotic lesions, including pulmonary fibrosis and wound healing, is the appearance of myofibroblasts at sites of wound healing.

Th17 cells have been identified as a subset of effector Th cells, and interleukin (IL)-17A is the major cytokine released from these or other IL-17-producing cells [4]. Our previous study [5] and other studies [6-8] have demonstrated a possible contribution for IL-17A in the development of pulmonary fibrosis.

It has been shown that NF- $\mathrm{kB}$ is involved in the mediation of IL-17 downstream signaling in various mammalian cell types. Such IL-17 signaling has been shown to be mediated through NF- $\kappa B$ activator 1 (Act1) and TNFR-associated factor 6 [9-11]. Act1 was recently shown to associate with both TNFR-associated factor 6 and IL-17R in fibroblasts [12]. These results indicate Act1 adaptor protein is not only an immediate and essential signaling component of the IL-17 receptor family, but also has implications in therapeutic treatment of various immune diseases.

Here, we explore the role of IL-17 in the proliferation, transformation and synthesis of collagen of lung fibroblasts, and the possible mechanism of the NF- $\mathrm{kB}$ signal pathway involved in this process of IL-17 on lung fibroblasts, and the function of siRNA-Act1 transfected into lung fibroblasts.

\section{Materials and Methods}

\section{Animals and Reagents}

C57BL/6 mice weighing 18-22 g were purchased from the experiment center of Kunming medical University; all animals were allowed to take food and tap water ad libitum. All procedures were in accordance with the Declaration of Helsinki of the World Medical Association. The protocols were also approved by the IRB/ Ethics Committee of Kunming Medical University. Bleomycin was purchased from Nippon Kayaku Co. (Tokyo, Japan) and all of the fluorescence-conjugated antibodies were obtained from eBioscience (San Diego, Calif., USA) and BD Biosciences (San Jose, Calif., USA). Antibodies against $\alpha$-smooth muscle actin (SMA), type I and type III collagen, p65, P-p65, transcription factor IkBa, phospho-IkBa and $\beta$-actin (Abcam, Cambridge, UK) were used to detect their respective antigens.

Model of Bleomycin-Induced Pulmonary Fibrosis and the Culture of Primary Lung Fibroblasts

After anesthesia with pentobarbital, bleomycin $(5 \mathrm{mg} / \mathrm{kg}$ ) was administered intratracheally in $50 \mu \mathrm{l}$ of saline to male C57BL/6 mice. On days 3, 7, 14 and 28 after bleomycin treatment, the animals were sacrificed and the lungs were removed intact ( $\mathrm{n}=5$ in each group). Primary lung fibroblasts were isolated from bleomycin-induced pulmonary fibrosis mice on days 14 according to the expression of IL-17RA. Mouse fibroblasts were isolated as described previously and used between passage three [13]. Briefly, fibroblasts were grown out and cultured in Dulbecco's modified Eagle medium (DMEM) with $4.5 \mathrm{~g} / \mathrm{l}$ of glucose supplemented with $10 \%$ heat-inactivated fetal bovine serum and $1 \%$ antibiotic-antimycotic solution $(100 \mathrm{U} / \mathrm{ml}$ penicillin G sodium, $100 \mathrm{U} / \mathrm{ml}$ streptomycin and $0.25 \mathrm{~g} / \mathrm{ml}$ amphotericin B). Identification of fibroblasts was based on the expression of vimentin.

\section{Proliferation Assay of MTT}

In brief, lung fibroblasts were seeded into flat-bottomed 96well microplates at a density of $5 \times 10^{3}$ cells $/ 0.2 \mathrm{ml}$ per well. After $24 \mathrm{~h}$, when the cells reached a subconfluent state, the cells were transferred to grow in a special culture medium containing various concentrations of recombinant IL-17 in an incubator at $37^{\circ} \mathrm{C}$ with $5 \% \mathrm{CO}_{2}$, up to $48 \mathrm{~h}$. The absorbance values of each well were determined spectrophotometrically at $490 \mathrm{nM}$ using a Microplate Reader (BIO-TEK, Rockville, Mass., USA). The best concentrations of IL-17 were selected, then the proliferation of lung fibroblasts through IL-17 stimulation were observed for 5 days following the above procedure.

\section{Immunofluorescence Staining}

For immunofluorescence microscopy, cells were rinsed with PBS and fixed with $2 \%$ formaldehyde in PBS for 3 min at room temperature. They were then permeabilized with $0.5 \%$ Triton X-100 (Sigma, St. Louis, Mo. USA) in PBS for $30 \mathrm{~min}$. This PBSTriton solution was also used for all subsequent antibody-washing steps. Primary antibody was used at an appropriate dilution for $1 \mathrm{~h}$ at $37^{\circ} \mathrm{C}$ in a humid chamber. Afterwards, stained samples were washed with washing solution three times, each for $10 \mathrm{~min}$. FITClabeled secondary antibodies (Santa Cruz Biotechnology, Santa Cruz, Calif., USA) were affinity-purified and tagged, and then counterstained with DAPI (Sigma) for the nuclear dye. Cell samples were examined with an epifluorescence microscope (Olympus) using filter sets that are selective for DAPI fluorescein. Color photographs were taken with an equipped digital camera and processed in Adobe Photoshop CS.

\section{Reverse-Transcription and Polymerase Chain Reaction}

IL-17 RmRNA expression in lung tissues was detected by RTPCR on days 3, 7, 14 and 28 after bleomycin infusion. Total RNA was isolated from lung homogenates with Trizol (Gibco-BRL, Gaithersburg, Md., USA). Lung fibroblasts were stimulated with IL-17 $(50 \mathrm{ng} / \mathrm{ml})$ and incubated for 24, 48 and $72 \mathrm{~h}$. The expression of $\alpha$-SMA, type I and type III collagen mRNA was detected. The reverse transcription reactions of the extracted RNA were performed by PCR in a final volume of $25 \mu \mathrm{l}(2 \mu \mathrm{l}$ of cDNA, $0.2 \mu \mathrm{mol} / \mathrm{l}$ of both forward and reverse primers, $1.5 \mathrm{mmol} / 1 \mathrm{MgCl}_{2}, 0.12 \mu \mathrm{mol} / \mathrm{l}$ and $180 \mu \mathrm{mol} / \mathrm{l} \mathrm{dNTP}$ in $1 \times$ PCR-Gold Buffer). The murine primers for PCR reactions were based on GenBank published sequences and are as follows:

type I collagen ( $5^{\prime}$-CGGCCCTGCTGGAAACCCTG-3'; $5^{\prime}$-GGGAGCACCACGTTCACCGG-3 $3^{\prime}$ ), type III collagen ( $5^{\prime}$-GCACCTGGAGAACGTGGGCC- $3^{\prime}$; $5^{\prime}$-GACCACGCCCACCGGGAAAG- $\left.3^{\prime}\right)$, a-SMA (5'-GCCAGTCGCTGTCAGGAACCCT-3'; $5^{\prime}$-G CCAGCCAAGTCCAGACGCAT- $\left.3^{\prime}\right)$, IL-17R ( $5^{\prime}$-CATCTGCGT- 
CTGACATAGTA-3'; $5^{\prime}$-CCTCTGTTCATTCTGGCTAA-3'), $\beta$ actin ( $5^{\prime}$-CTGGCCGGGACCTGACAGACT- $3^{\prime} ; 5^{\prime}$-AGAAAGGGTGTAAAACGCAGCTCA-3').

Western Blotting Analysis

Cells were washed twice with ice-cold PBS and ruptured with lysis buffer containing $20 \mathrm{mmol} / \mathrm{l} \mathrm{Tris}-\mathrm{HCl}, 150 \mathrm{mmol} / \mathrm{l} \mathrm{NaCl}, 1 \%$ Triton X-100, inhibitors of protease and phosphatase. Cell extracts were microcentrifuged for $20 \mathrm{~min}$ at $10,000 \mathrm{~g}$ and supernatants were collected. In all, $30 \mu \mathrm{g}$ of cellular protein was resolved by SDS-PAGE and transferred onto PVDF membranes. The membranes were blocked for $1 \mathrm{~h}$ with 5\% skimmed milk in Trisbuffered saline containing $0.1 \%$ Tween 20 and incubated overnight at $4^{\circ} \mathrm{C}$ with primary antibodies. Membranes were washed, incubated for $1 \mathrm{~h}$ with appropriate secondary antibodies, conjugated to horseradish peroxidase and their immunoreactivity was visualized by chemiluminescence. The scanned figures were visualized and quantified using Image J software.

\section{Knockdown of Act1}

siRNAs for Act1 ( $5^{\prime}$-GUCAAUUGCUGAAACCGAU-3'; Santa Cruz Biotechnology) and control scrambled siRNAs (Santa Cruz Biotechnology) were transfected into lung fibroblasts at a final concentration of 25 pmol using the Lipofectamine 2000 (Invitrogen, Carlsbad, Calif., USA). After transfection for $24 \mathrm{~h}$, cells were stimulated with IL-17 (50 ng/ml) for $48 \mathrm{~h}$ or 15,30 or $60 \mathrm{~min}$ and harvested for semiquantitative real-time PCR and Western blot analysis as described earlier.

\section{Statistical Analysis}

Western blotting and RT-PCR experiments were performed in triplicate. Data are expressed as mean \pm SD. Statistical significance was analyzed with one-way analysis of variance with pairwise contrasts by Scheffe's method. Differences were assumed to be statistically significant when $\mathrm{p}$ values were less than 0.05 .

\section{Results}

\section{IL-17 Promoted the Proliferation and Collagen}

\section{Synthesis of Lung Fibroblasts}

We successfully constructed a pulmonary fibrosis model after airway bleomycin administration. In order to elucidate the mechanism of IL-17 in pulmonary fibrosis, lung fibroblasts were selected as targets of study. We verified the expression of IL-17R in bleomycin-induced pulmonary fibrosis. According to the expression of IL-17R trend (fig. 1a), lung tissues at 14 days were selected, extracted and cultured for primary lung fibroblasts (fig. 1b). In order to ascertain the best concentration of IL-17, various concentrations $(25,50,100$ and $200 \mathrm{ng} / \mathrm{ml})$ were cocultured with lung fibroblasts for $48 \mathrm{~h}$. The proliferation of lung fibroblasts in response to IL-17 treatment by a dose-dependent method was found, and $50 \mathrm{ng} / \mathrm{ml}$ of IL-17 most obviously promoted the proliferation of lung fibroblasts, leading to its selection as the best concentration. Therefore, when ex vivo lung fibroblasts were incubated with IL-17 $(50 \mathrm{ng} / \mathrm{ml})$ in serum-free conditions for 5 days of continuous observation, the results showed that the proliferation of lung fibroblast was significantly changed on day 2 (fig. 1c, d). At the same time, when type I and type III collagen were measured by RT-PCR and Western blot for 24, 48 and $72 \mathrm{~h}$, the results demonstrated that IL-17 promoted the collagen synthesis of lung fibroblasts (fig. 1e, f).

\section{IL-17 May Promote the Transformation of Lung Fibroblast to Myofibroblast}

At the same time, we verified the effects of IL-17 on myofibroblast transformation as demonstrated by its ability to induce expression of a-SMA, a well-known marker of myofibroblast transdifferentiation. Immunofluorescence staining of fibroblasts also revealed that IL-17 stimulated the organization of a-SMA into the structure of the cytoskeleton (fig. 2a). RT-PCR analysis revealed that IL-17 induced $\alpha$-SMA mRNA expression in a time-dependent manner. Similarly, IL-17-treated primary lung fibroblasts displayed the expression of $\alpha$-SMA at the transcript and protein level (fig. 2b, c).

\section{Increased Levels of NF- $\kappa B$ Pathway Following IL-17}

\section{Stimulation}

We found that IL-17 stimulation on lung fibroblasts did not induce the increase of p65 and Ikb protein through Western blot, but higher levels of basal phosphorylated p65 and Ikb were significantly exhibited with incubation of IL-17 (50 ng/ml) for $5 \mathrm{~min}$ (fig. 3a). The SEFIR domain in both IL-17RA and Act1 is required for IL-17-induced NF- $\kappa B$ activation. To investigate the roles of Act1 in IL-17-dependent signaling, we examined the expression of Act 1 in lung fibroblasts. As shown in figure 3a, lung fibroblasts expressed protein for Act1, but IL-17 did not increase the expression of Act 1. In order to ascertain the role of Act1 on lung fibroblasts for IL-17, we knocked down Act1 expression using liposome-mediated siRNA. In addition, IL-17-induced expression of P-p65 and p-Iкb were significantly lower in lung fibroblasts of the silencing of Act1 (fig. 3b).

\section{The Silencing of Act1 Inhibited the Role of IL-17 on the} Lung Fibroblasts

We examined the role of IL-17-induced proliferation, transformation and synthesis of collagen in lung fibroblasts of the knockdown of Act1 and found that the role of the IL-17-induced proliferation was inhibited by MTT 


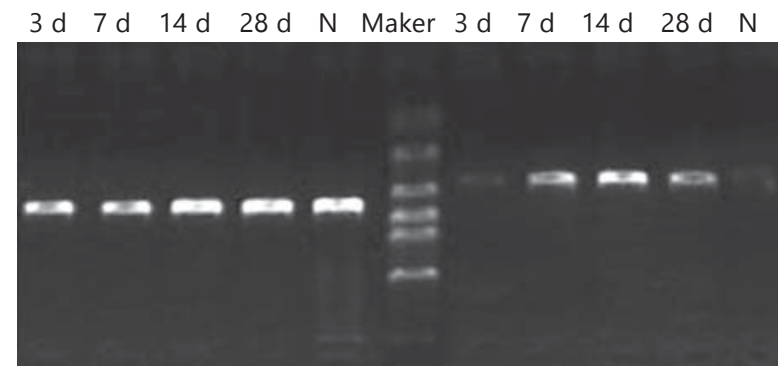

$\beta$-actin

IL-17R

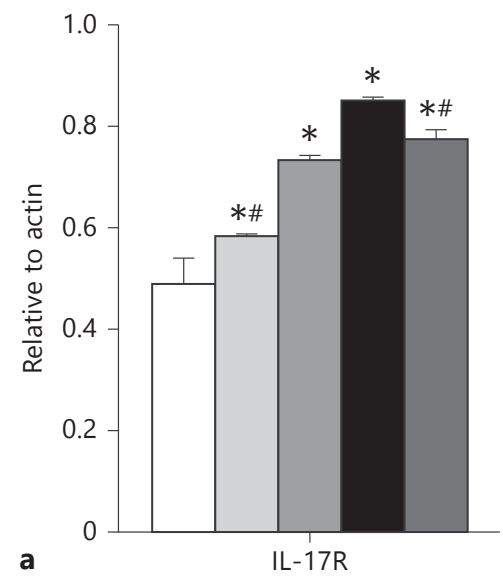

$\square$ Control

$\square 3 \mathrm{~d}$

$\square 7 \mathrm{~d}$

- $14 \mathrm{~d}$

$\square 28 \mathrm{~d}$ a

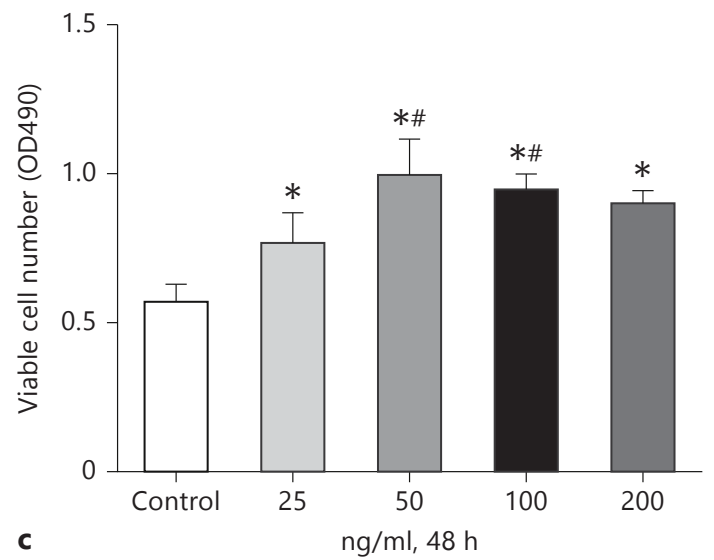

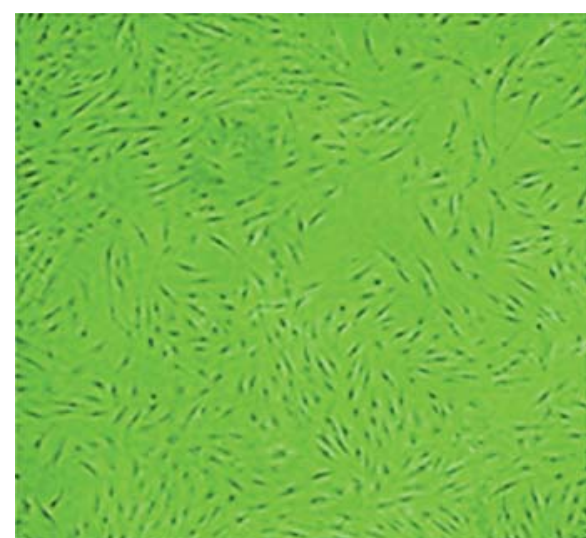
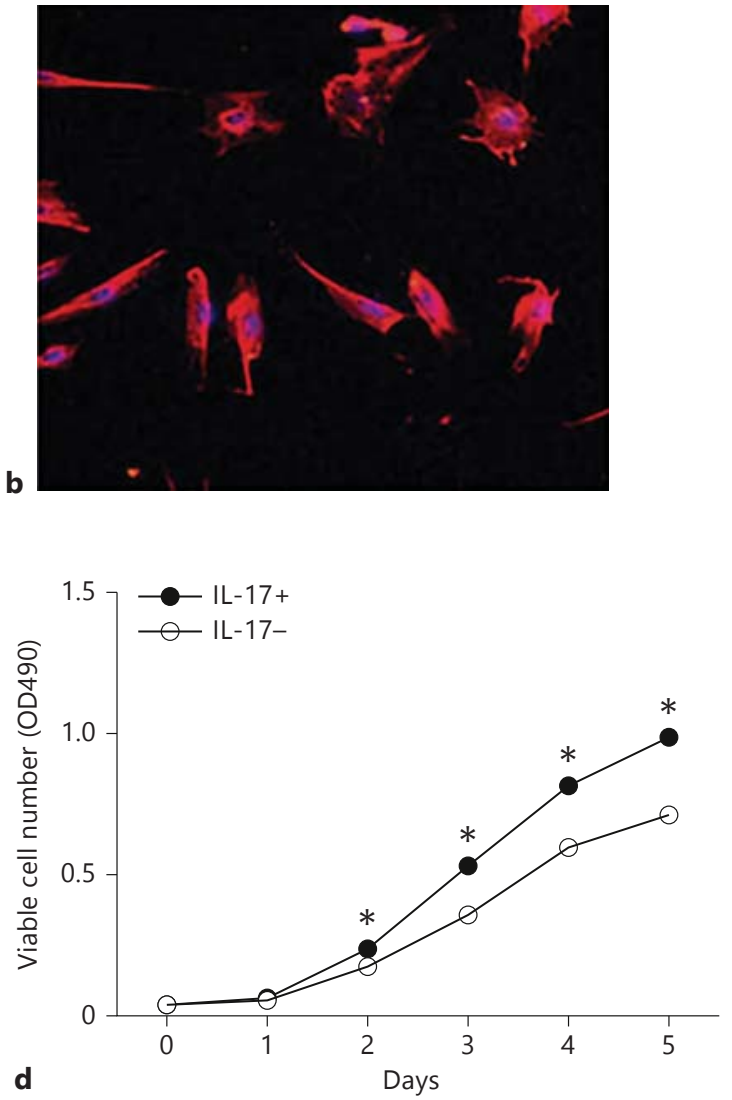

Fig. 1. IL-17 promoted the proliferation and collagen synthesis of lung fibroblasts. A pulmonary fibrosis model was induced by airway $5 \mathrm{mg} / \mathrm{kg}$ bleomycin administration, and sacrificed on days (d) 3, 7, 14 and 28. a The expression of IL-17R in bleomycininduced pulmonary fibrosis was measured by RT-PCR. b Identification of lung fibroblasts was done by light microscope $(\times 100)$ and laser scanning confocal microscope through labeled vimentin (positive cells were red in cytoplasm). c The primary lung fibroblasts were exposed to IL-17 at different concentra- tions, 25, 50, 100 and $200 \mathrm{ng} / \mathrm{ml}$ for $48 \mathrm{~h}$, and the proliferation role of IL-17 on lung fibroblast was measured. * $\mathrm{p}<0.05$ compared with controls, ${ }^{*} \mathrm{p}<0.05$ compared with $25 \mathrm{ng} / \mathrm{ml}$. The proliferation role at $50 \mathrm{ng} / \mathrm{ml}$ concentration of IL-17 was observed for 5 days (d), lung fibroblasts were incubated with IL-17 $(50 \mathrm{ng} / \mathrm{ml})$ for 24,48 and $72 \mathrm{~h}$, and RNA and protein expression of type I collagen (ColI) and type III collagen (ColIII) was determined by RT-PCR and Western blot $(\mathbf{e}, \mathbf{f}){ }^{*} \mathrm{p}<0.05$ compared with controls. 

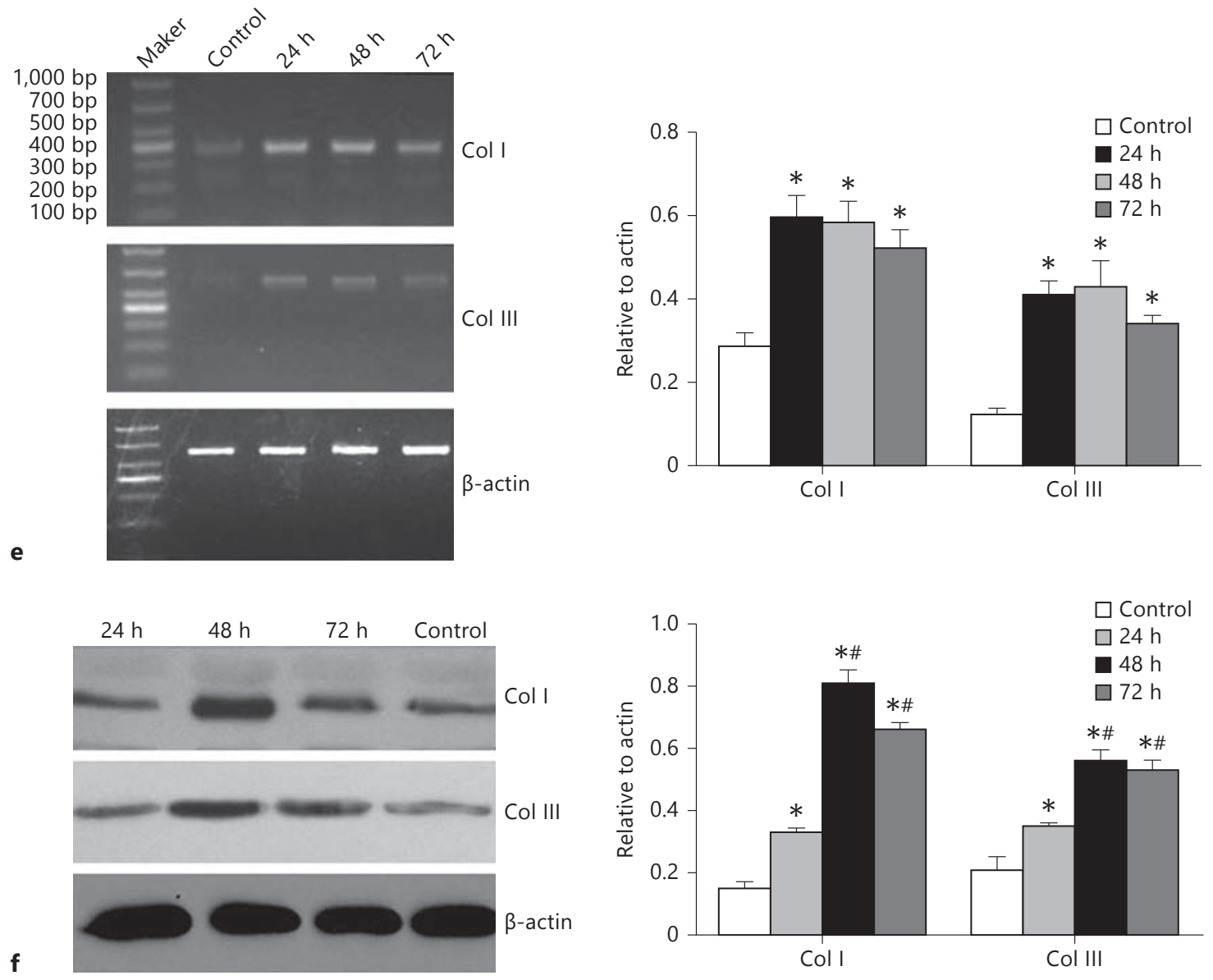

(fig. 4c). Similarly, knockdown of Act1 reduced the IL17-dependent transformation of lung fibroblasts through immunofluorescence staining at $48 \mathrm{~h}$ (fig. 4a), the expression of $\alpha$-SMA protein was decreased by Western blot, and the synthesis of type I and III collagen was inhibited (fig. 4b, d, e).

\section{Discussion}

Current understanding of the pathogenesis of pulmonary fibrosis suggests that fibroblasts and myofibroblasts play an important role in the diverse processes that constitute fibrosis. Lung fibroblasts are the main effective cells of pulmonary fibrosis, which can not only be transformed into myofibroblasts, but can also promote the synthesis of collagen and secretion of cytokines in the progress of pulmonary fibrosis.
IL-17A directly modulates fibroblast inflammatory functions by increasing IL-6, IL-8, G-CSF and matrix metalloproteinase production [14-16]. In vitro studies have also shown that rIL-17A did or did not upregulate the proliferation and collagen expression of cardiac or skin fibroblasts, respectively [17, 18]. Lo Re et al. [19] found that rmIL-17A, but not rmIL-22, significantly stimulated lung fibroblast proliferation only in cultures obtained from silica-treated mice. Contrary to rmTGF-b1, Th17 cytokines did not induce collagen or fibronectin expression or myofibroblast differentiation.

Lung fibroblasts in a bleomycin-induced pulmonary fibrosis model were extracted and cultured in this study, and acted as targets of IL-17 directly. The results found that IL17 could directly promote the transformation of lung fibroblasts into myofibroblasts and the synthesis of collagen.

A number of reports have indicated that activation of NF-kB is a major mechanism by which IL-17 regulates 


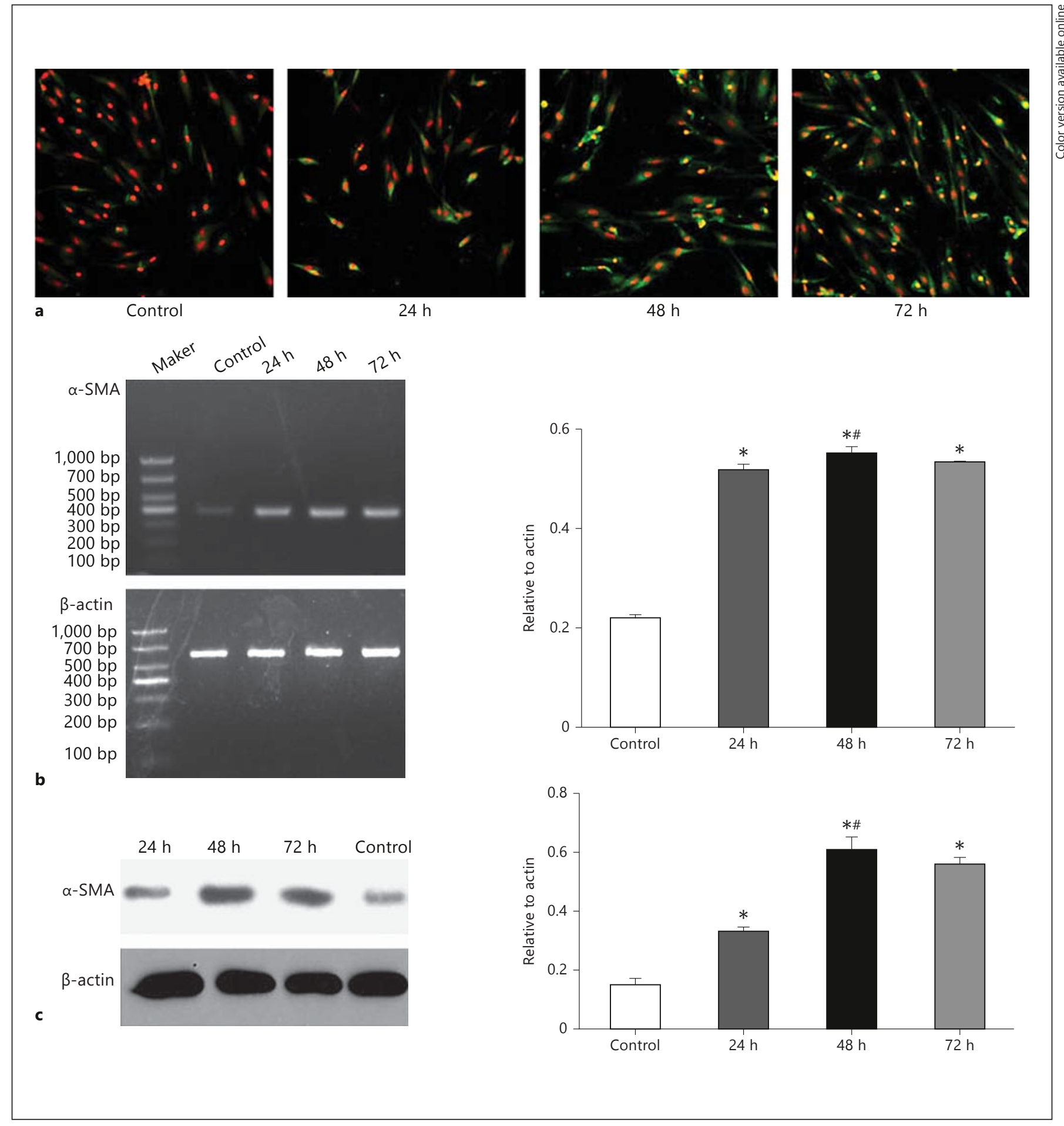

Fig. 2. The IL-17 may promote the transformation of lung fibroblast to myofibroblast. a Immunofluorescence microscopy was used to assess the degree of $\alpha$-SMA (green) and cell nucleus

(red) for 24, 48 and 72 h. b, c RNA and protein of $a$-SMA was determined by RT-PCR and Western blot. ${ }^{*} \mathrm{p}<0.05$ compared with controls, ${ }^{\#} \mathrm{p}<0.05$ compared with $24 \mathrm{~h}$. 


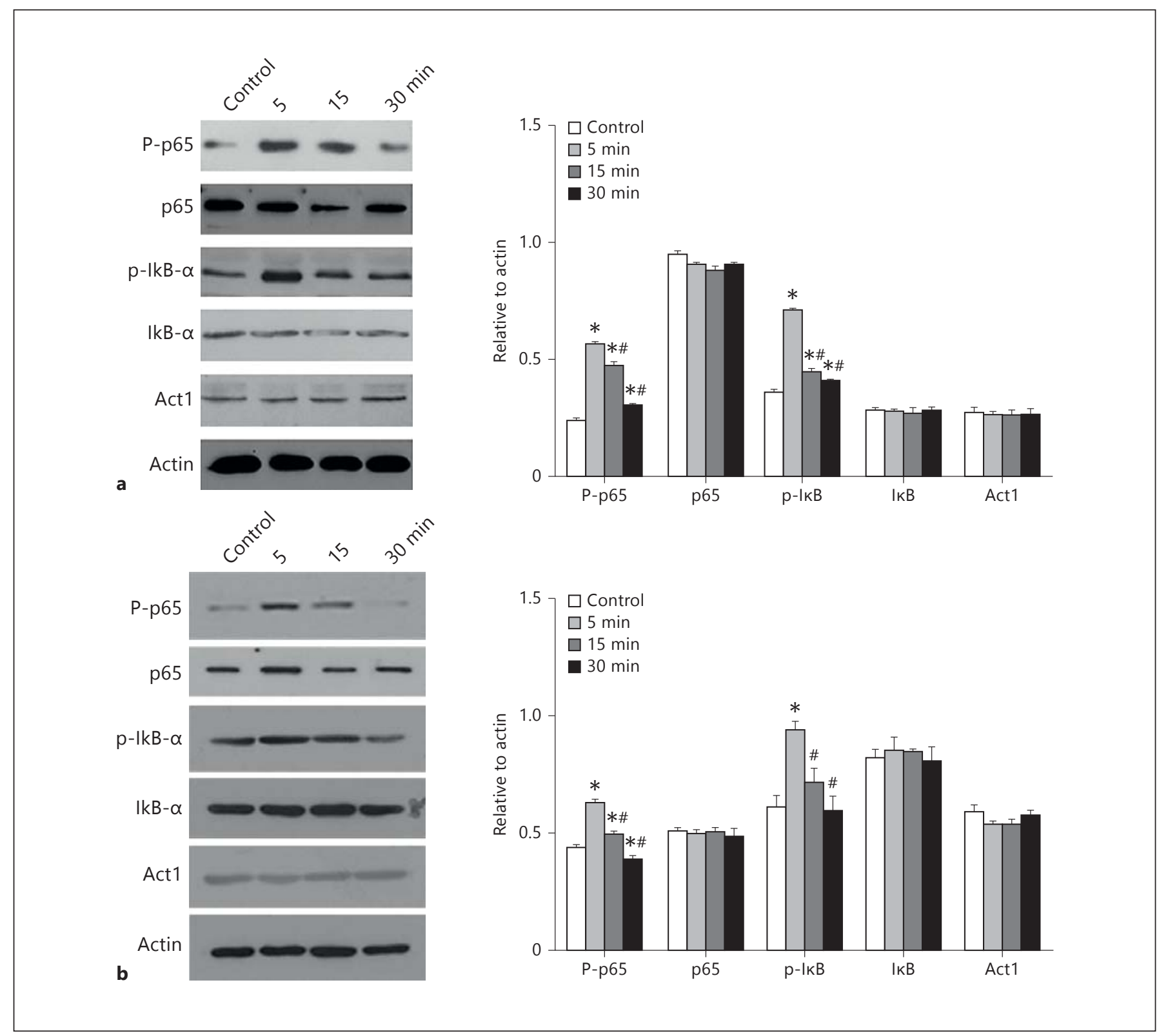

Fig. 3. Increased levels of NF- $\kappa$ B pathway following IL-17 stimulation. a Lung fibroblasts were treated with IL-17 (50 ng/ml) and cells were harvested at the indicated time points. The levels of the indicated protein were analyzed by Western blot analysis with the respective antibodies. Quantitation of the indicated protein after normalization to the levels of $\beta$-actin is graphed

target gene expression. Act1 adaptor protein is an immediate and essential signaling component of IL-17 receptor [9].The importance of Act1 in IL-17 signaling has been demonstrated by various disease models in the mouse [20]. We demonstrated the activation of P-p65 as the mean $\pm \mathrm{SD}$. b Lung fibroblasts were transfected with siRNA-Actl. After $24 \mathrm{~h}$ of siRNA transfection, the cells were treated with IL-17 (50 ng/ml). Western blot analysis determined the levels of protein in the siRNA-transfected lung fibroblasts. ${ }^{*} \mathrm{p}<0.05$ compared with controls, ${ }^{*} \mathrm{p}<0.05$ compared with 5 min. 


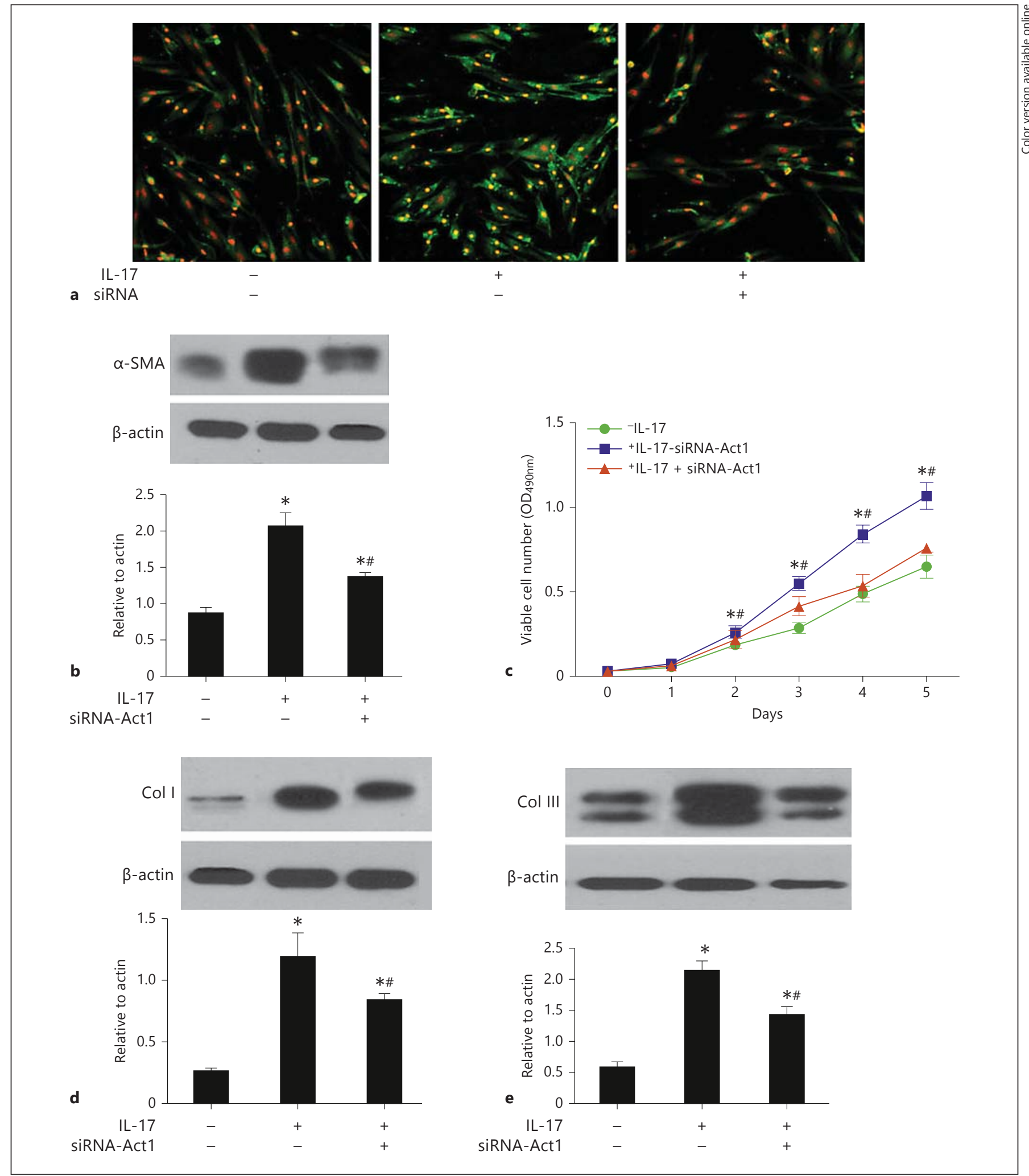

Fig. 4. The silencing of Act 1 inhibited the role of IL-17 on the lung fibroblasts. a Immunofluorescence microscopy was used to assess the degree of a-SMA (green) and cell nucleus (red) after 48 h. b, d, e Protein of $\alpha$-SMA, type I collagen (ColI) and type III collagen (ColIII) were determined by RT-PCR and Western blot. c The proliferation of IL-17 on lung fibroblasts was measured by MTT. 
sion of P-p65 and p-IkB. Deficiency of Act1 in lung fibroblasts leads to impair of the proliferation and transformation of lung fibroblasts into myofibroblasts and collagen synthesis upon IL-17 treatment.

In conclusion, this study has demonstrated that IL-17 can directly promote proliferation, transformation and collagen synthesis of lung fibroblast. Silencing Act1 may decrease this function of IL-17 through the NF- $\mathrm{kB}$ pathway, and strategies that specifically block this pathway may provide the next generation of efficacious therapies.

\section{Acknowledgements}

This work was supported by the Natural Science Foundation of Yunnan Province Science and Technology Department (No. 2009ZC107M) and the Key Project for Science Research Fund of Yunnan Province Education Department (No. 2010Z029).

\section{Financial Disclosure and Conflicts of Interest}

The authors declare that they have no competing interests.

\section{References}

1 Gharaee-Kermani M, Phan SH: Molecular mechanisms of and possible treatment strategies for idiopathic pulmonary fibrosis. Curr Pharm Des 2005;11:3943-3971.

2 Wilson MS, Wynn TA: Pulmonary fibrosis: pathogenesis, etiology and regulation. Mucosal Immunol 2009;2:103-121.

-3 Strieter RM, Mehrad B: New mechanisms of pulmonary fibrosis. Chest 2009;136:13641370.

4 Chen Z, O'Shea JJ: Th17 cells: a new fate for differentiating helper $\mathrm{T}$ cells. Immunol Res 2008;41:87-102.

5 Dong Z, Tai W, Yang Y, et al: The role of alltrans retinoic acid in bleomycin- induced pulmonary fibrosis in mice. Exp Lung Res 2012; 38:82-89.

6 Roark CL, Simonian PL, Fontenot AP, Born WK, O'Brien RL: $\gamma \delta$ T cells: an important source of IL-17. Curr Opin Immunol 2008;20: 353-357.

7 Braun RK, Ferrick C, Neubauer P, et al: IL-17 producing $\gamma \delta$ T cells are required for a controlled inflammatory response after bleomycin-induced lung injury. Inflammation 2008; 31:167-179.

8 Simonian PL, Roark CL, Wehrmann F, et al: Th17-polarized immune response in a murine model of hypersensitivity pneumonitis and lung fibrosis. J Immunol 2009;182:657-665. $\checkmark$ Chang SH, Park H, Dong C: Act1 adaptor protein is an immediate and essential signaling component of interleukin-17 receptor. J Biol Chem 2006;281:35603-35607.

10 Li X: Act1 modulates autoimmunity through its dual functions in CD40L/BAFF and IL-17 signaling. Cytokine 2008;41:105-113.

11 Shalom-Barak T, Quach J, Lotz M: Interleukin-17-induced gene expression in articular chondrocytes is associated with activation of mitogen-activated protein kinases and NF-kB. J Biol Chem 1998;273: 27467-27473.

12 Park H, Li Z, Yang XO, et al: A distinct lineage of CD4 T cells regulates tissue inflammation by producing interleukin 17 . Nat Immunol 2005;6:1133-1141.

13 Ramirez AM, Wongtrakool C, Welch T, et al: Vitamin D inhibition of pro-fibrotic effects of transforming growth factor $\beta 1$ in lung fibroblasts and epithelial cells. J Steroid Biochem Mol Biol 2010;3:142-150.

14 Fossiez F, Djossou O, Chomarat P, et al: T cell interleukin-17 induces stromal cells to produce proinflammatory and hematopoietic cytokines. J Exp Med 1996;183:25932603.
15 Hwang SY, Kim JY, Kim KW, et al: IL-17 induces production of IL- 6 and IL- 8 in rheumatoid arthritis synovial fibroblasts via NF- $\mathrm{kB}$ and PI3-kinase/Akt-dependent pathways. Arthritis Res Ther 2004;6:R120-R128.

16 Bamba S, Andoh A, Yasui H, et al: Matrix metalloproteinase-3 secretion from human colonic subepithelial myofibroblasts: role of interleukin-17. J Gastroenterol 2003;38:548554.

17 Venkatachalam K, Mummidi S, Cortez DM, et al: Resveratrol inhibits high glucose-induced PI3K/Akt/ERK-dependent interleukin-17 expression in primary mouse cardiac fibroblasts. Am J Physiol Heart Circ Physiol 2008;294:H2078-H2087.

18 Kurasawa K, Hirose K, Sano H, et al: Increased interleukin-17 production in patients with systemic sclerosis. Arthritis Rheum 2000;43: 2455-2463.

19 Lo ReS, Dumoutier L, Couillin I, et al: IL-17Aproducing $\gamma \delta \mathrm{T}$ and Th17 lymphocytes mediate lung inflammation but not fibrosis in experimental silicosis. J Immunol 2010;184: 6367-6377.

20 Qian Y, Liu C, Hartupee J, et al: The adaptor Act1 is required for interleukin 17-dependent signaling associated with autoimmune and inflammatory disease. Nat Immunol 2007;8: 247-256. 